Results Median (IQR) sufentanil central and total volume of distribution, clearance, and distribution and elimination halflife were $4.7(4.2-5.2) \mathrm{L} / \mathrm{kg}$ and $11.8(9.9-14.2) \mathrm{L} / \mathrm{kg}, 0.552$ (0.415- 0.671) L/h/kg, and $0.0264(0.0260-0.0264) \mathrm{h}$ and 15.7 (13.4-19.2) h, respectively.

Conclusion We observed similar sufentanil PK parameters (preliminary results) as described previously in literature. However, further studies with more patients are needed.

Funding This study is supported by a Charles University project Progres Q25, SVV 260373 and a project MH CZ-DRO VFN64165, and by an unrestricted research grant of the Intensive Care of the ErasmusMC-Sophia Children's Hospital. Disclosure(s) Nothing to disclose

\section{P80 OBJECTIVE PHARMACODYNAMIC EVALUATION OF DOXAPRAM IN PRETERM INFANTS}

${ }^{1} \mathrm{JA}$ Poppe*, ${ }^{2} \mathrm{~W}$ van Weteringen, ${ }^{3} \mathrm{~S}$ Völler, ${ }^{1} \mathrm{TG}$ Goos, ${ }^{1} \mathrm{IKM}$ Reiss, ${ }^{1} \mathrm{SHP}$ Simons ${ }^{1}$ Department of Paediatrics, Division of Neonatology, Erasmus MC - Sophia Children's Hospital; ${ }^{2}$ Department of Paediatric Surgery, Erasmus MC - Sophia Children's Hospital, Rotterdam; ${ }^{3}$ Division of Pharmacology, Leiden Academic Centre for Drug Research, Leiden University, Leiden, The Netherlands

\subsection{6/archdischild-2019-esdppp.118}

Background Pharmacodynamic evaluation is challenging in neonatal clinical care, and often based on subjective human interpretation of clinical signs and the available 'snapshot' of physiological parameters. High frequency data $(\geq 1 \mathrm{~Hz})$ of bedside patient monitors provide the opportunity of continuous and objective drug evaluation. ${ }^{1}$ This study investigates the predictive value of continuously available vital and ventilatory parameters to evaluate doxapram therapy. Doxapram is a respiratory stimulant to avoid mechanical ventilation and adverse outcomes of hypoxemia in preterm infants. ${ }^{2}$

Methods Preterm infants admitted to a level III NICU centre who received doxapram therapy were eligible for inclusion. Stored vital and ventilatory parameters were retrospectively analysed. Multivariate analysis was performed to identify variables that influenced therapy failure (intubation or death) or success. Variables with a $p$ value $<0.1$ in univariate analysis were included in the multivariate analysis. Additionally, the $\Delta \mathrm{SpO}_{2} / \mathrm{FiO}_{2}$-ratio was calculated by subtracting the median $\mathrm{SpO}_{2} / \mathrm{FiO}_{2}$-ratio 1 day after therapy from the median $\mathrm{SpO}_{2} /$ $\mathrm{FiO}_{2}$-ratio 1 day before therapy.

Results The first episode of doxapram treatment was analysed in a total of 61 preterm infants with a median postnatal age at therapy start (PNA) of 3.0 weeks (IQR; 2.0-3.6). The success rate of doxapram therapy was $57 \%$. Out of all parameters, the $\mathrm{SpO}_{2} / \mathrm{FiO}_{2}$-ratio showed to be the most indicative for therapy outcome. The predictive model included the $\Delta \mathrm{SpO}_{2} /$ $\mathrm{FiO}_{2}$-ratio, the PNA, the administration of a loading dose at start, and intubation within 24 hours before the start of doxapram (area under the curve of 0.828 ). The $\Delta \mathrm{SpO}_{2} / \mathrm{FiO}_{2}$-ratio was inversely associated with therapy outcome (OR 0.26 , CI 95\% 0.07-0.83; $p=0.03$ ).

Conclusion The $\Delta \mathrm{SpO}_{2} / \mathrm{FiO}_{2}$-ratio between 1 day before and 1 day after start of the therapy is predictive of failure or success of doxapram therapy. The use of physiological data shows potential in the pharmacodynamic evaluation of doxapram therapy.

\section{REFERENCES}

1. Flint $R$, Weteringen WV, Voller $S$, Poppe JA, Koch BC, Groot R, et al. Big data analyses for continuous evaluation of pharmacotherapy: A proof of principle with doxapram in preterm infants. Curr Pharm Des. 2017.

2. Poets CF, Roberts RS, Schmidt B, Whyte RK, Asztalos EV, Bader D, et al. Association Between Intermittent Hypoxemia or Bradycardia and Late Death or Disability in Extremely Preterm Infants. JAMA. 2015;314(6):595-603.

Disclosure(s) Nothing to disclose

\section{P81 USE OF SYSTEMIC ANTIBIOTICS IN AUSTRIAN CHILDREN AND ADOLESCENTS: A SURVEY}

${ }^{1} \mathrm{E}$ Rauch ${ }^{*},{ }^{2} \mathrm{FB}$ Lagler, ${ }^{3} \mathrm{H}$ Herkner, ${ }^{4} \mathrm{~V}$ Strenger, ${ }^{1} \mathrm{C}$ Male. ${ }^{1}$ Department of Paediatric and Adolescent Medicine, Medical University Vienna, Vienna; Institute for Inborn Errors of Metabolism and Department of Paediatrics, Paracelsus Medical University, Salzburg; ${ }^{3}$ Department of Emergency Medicine, Medical University Vienna, Vienna; ${ }^{4}$ Department of Paediatric Haemato-Oncology, Medical University Graz, Graz, Austria

\subsection{6/archdischild-2019-esdppp.119}

Background Several surveys that have assessed the use of antibiotic medicines for children and adolescents demonstrate significant heterogeneity in prescription patterns between different countries. The liberal use of antibacterial treatment, particularly unjustified use of broad spectrum antibiotics, is one of the main reasons for increasing rates of antibiotic resistance. We have studied antibiotics prescription frequencies in children and adolescents in Austria covering both primary and hospital care settings, in order to obtain a representative survey for our country.

Methods Prescription data for systemic antibiotics were assessed for the year 2014. For primary care, reimbursement data were obtained from Austrian health insurances. For hospital care, information on medicines dispensed to paediatric wards was obtained from hospital pharmacies. Frequencies of systemic antibiotic use were analyzed by Anatomical Therapeutic Chemical classification system, age groups, care setting, and in relation to all other medicines used. Systemic antibiotics were divided into subgroups, like broad and narrow spectrum penicillins, cephalosporins, tetracyclins, and others.

Results Systemic antibiotics comprised 24\% and 21\% of all medicines prescribed to children and adolescents in primary care and hospital care, respectively. The most frequent were broad spectrum antibiotics $32 \%$ and $35 \%$ of systemic antibiotics prescribed) in both primary and hospital care settings. Macrolides and 2nd generation cephalosporins were more frequently prescribed in primary care setting, whereas B-lactam antibiotics other than cephalosporins and antibiotics, such as fluorochinolones, aminoglycosides, or linezolid were more frequently used in hospital.

Conclusions This is the first representative survey investigating the use of systemic antibiotics in children and adolescents in Austria. The study allows comparison of prescription patterns to other European countries: it reveals rather big differences to systemic antibiotics prescription in Italy, the Netherlands and Denmark, whereas prescription patterns are similar in Germany and Austria. Furthermore, this survey provides the basis to assess temporal trends in the future.

Disclosure(s) Nothing to disclose 